PROCEEDINGS OF THE

AMERICAN MATHEMATICAL SOCIETY

Volume 132, Number 12, Pages 3513-3523

S 0002-9939(04)07512-4

Article electronically published on June 2, 2004

\title{
THE STRONG NO LOOP CONJECTURE FOR SPECIAL BISERIAL ALGEBRAS
}

\author{
SHIPING LIU AND JEAN-PHILIPPE MORIN
}

(Communicated by Martin Lorenz)

Dedicated to Claus M. Ringel on the occasion of his 60 th birthday

ABSTRACT. We establish the strong no loop conjecture for some special cases, in particular, for special biserial algebras.

\section{INTRODUCTION}

Let $A$ be a finite-dimensional algebra over a field given by a quiver with relations. Let $S$ be a simple $A$-module with a non-split self-extension; that is, the quiver has a loop at the corresponding vertex. The strong no loop conjecture claims that $S$ is of infinite projective dimension; see [1, 6]. This conjecture remains open except for monomial algebras; see, for example, 2, 6, 8, 11. Under certain hypotheses on the loop, Green, Solberg and Zacharia have shown that $\operatorname{Ext}_{A}^{i}(S, S)$ does not vanish for every $i \geq 1$; see [5, (4.2)]. In this paper, we shall first present a short proof of this result, because not only is the original proof rather complex, but also our idea possibly works for other cases. Next we observe that this result reduces the conjecture to the case where some power of the loop is a component of a polynomial relation. This reduction works particularly well when $A$ is special biserial, due to a combinatorial description of the syzygies of string modules; see (2.2). Our main result says that $\operatorname{Ext}_{A}^{i}(S, S)$ does not vanish for every $i \geq 1$ if the convex support of $S$ is special biserial. We shall also prove that if $S$ has an almost split self-extension, then the block of $A$ supporting $S$ is a local Nakayama algebra; in particular, $\operatorname{Ext}_{A}^{i}(S, S)$ does not vanish for every $i \geq 1$. In the course of its proof, we easily get a characterization of Nakayama algebras, strengthening the one stated in [1, (IV.2.10)]. Contrary to what will be seen in this paper, Happel's example stated in [5, Section 4] shows the existence of a simple module $S$ with a loop but $\operatorname{Ext}^{i}(S, S)=0$ for infinitely many $i$. Our motivation for studying special biserial algebras comes from the following two aspects. First of all, since their representations are completely understood, they form naturally a testing class for various well-known conjectures in the representation theory of algebras. Secondly, these algebras play an important role in the modular representation theory of finite groups; see [7, tracing back to the classification of the indecomposable HarishChandra modules of the Lorentz group by Gelfand and Ponomarev; see [4].

Received by the editors April 22, 2003 and, in revised form, May 10, 2003 and September 4, 2003.

2000 Mathematics Subject Classification. Primary 16E05. 


\section{LOOPS WITH NO POWER A COMPONENT OF POLYNOMIAL RELATIONS}

To begin with, we fix some notation and terminology. Throughout this paper, $k$ stands for a field and $Q$ for a finite quiver. Let $k Q$ be the path algebra of $Q$ over $k$, and $Q^{+}$the ideal generated by the arrows. Note that we shall compose the paths of $Q$ from the left to the right. If $I$ is an ideal such that $\left(Q^{+}\right)^{m} \subseteq I \subseteq\left(Q^{+}\right)^{2}$ for some $m \geq 2$, then the pair $(Q, I)$ is called a bound quiver. We shall always assume that $I$ is such an ideal. Let $p_{1}, \ldots, p_{r}$ be pairwise distinct paths of $Q$ from a vertex $a$ to a vertex $b$, and let $\lambda_{1}, \ldots, \lambda_{r} \in k$ be nonzero scalars. We call

$$
\rho=\lambda_{1} p_{1}+\cdots+\lambda_{r} p_{r}
$$

a relation on $Q$ if $\rho \in I$ while $\sum_{i \in \Omega} \lambda_{i} p_{i} \notin I$ for all $\Omega \subset\{1, \ldots, r\}$. In this case, $p_{1}, \ldots, p_{r}$ are called the components of $\rho$ and $a$ the start-point. Moreover, $\rho$ is called monomial, binomial, or polynomial if $r=1, r=2$, or $r \geq 2$, respectively.

The quotient $A=k Q / I$ is called the algebra of the bound quiver $(Q, I)$. If $x \in k Q$, we shall denote by $\bar{x}$ the class $x+I \in A$. An $A$-module means a right module of finite $k$-dimension except otherwise stated explicitly. The radical, the top, and the $n$-th syzygy of an $A$-module $M$ will be denoted by $\operatorname{rad} M, \operatorname{top} M$ and $\Omega^{n}(M)$, respectively. We call $x \in M$ a top element if $x \notin \operatorname{rad} M$. For a vertex $a$ of $Q$, we shall denote by $S(a)$ and $P(a)$ the simple $A$-module and the indecomposable projective $A$-module associated to $a$, respectively.

The elements of a direct sum of $A$-modules are written as column matrices. Let $e_{1}, \ldots, e_{r}$ be idempotents of $A$. Then an $A$-linear map $\phi: e_{1} A \oplus \cdots \oplus e_{r} A \rightarrow M$ is left multiplication by a matrix $\left(x_{1}, \ldots, x_{r}\right)$ with $x_{i} \in M e_{i}$. In this case, we say that $\phi$ is represented by $\left(x_{1}, \ldots, x_{r}\right)$. In particular, if $f_{1}, \ldots, f_{s}$ are also idempotents of $A$, then an $A$-linear map from $e_{1} A \oplus \cdots \oplus e_{r} A$ to $f_{1} A \oplus \cdots \oplus f_{s} A$ is represented by an $(s \times r)$-matrix whose $(i, j)$-entry is an element of $f_{i} A e_{j}$. For convenience of reference, we state the following well-known result.

1.1. Lemma. Let $A$ be the algebra of a finite bound quiver with $e_{1}, \ldots, e_{s}$ some primitive idempotents. Let $M$ be a non-zero $A$-module with $x_{i} \in M e_{i}$ for $1 \leq i \leq s$. If the classes of $x_{1}, \ldots, x_{s}$ in top $M$ are linearly independent over $k$, then there exist primitive idempotents $e_{s+1}, \ldots, e_{r} \in A$ and $x_{s+1} \in M e_{s+1}, \ldots, x_{r} \in M e_{r}$ such that

$$
\left(x_{1}, \ldots, x_{s}, x_{s+1}, \ldots, x_{r}\right): e_{1} A \oplus \cdots \oplus e_{s} A \oplus e_{s+1} A \oplus \cdots \oplus e_{r} A \longrightarrow M
$$

is a projective cover of $M$.

We now give the promised alternative proof of Proposition 4.2 in [5].

1.2. Proposition (Green-Solberg-Zacharia). Let $A$ be the algebra of a bound quiver $(Q, I)$, containing a loop $\alpha$ at a vertex a. If for some $n \geq 2, \alpha^{n}$ lies in $I$ but not in $I Q^{+}+Q^{+} I$, then for all $i \geq 1, \operatorname{Ext}_{A}^{i}(S(a), S(a))$ does not vanish.

Proof. Suppose that $\alpha^{n} \in I$ but $\alpha^{n} \notin I Q^{+}+Q^{+} I$. In particular, $\alpha^{n-1} \notin I$. Let $\alpha_{i}: a \rightarrow c_{i}, i=1, \ldots, t$, be the arrows starting at $a$ with $\alpha_{1}=\alpha$. Then $S(a)$ admits a minimal projective resolution

$$
\cdots \longrightarrow P_{m} \stackrel{\phi_{m}}{\longrightarrow} P_{m-1} \longrightarrow \cdots \longrightarrow P_{1} \stackrel{\phi_{1}}{\longrightarrow} P_{0} \longrightarrow S(a) \longrightarrow 0
$$

where $P_{0}=P(a), P_{1}=P\left(c_{1}\right) \oplus \cdots \oplus P\left(c_{t}\right)$, and $\phi_{1}$ is represented by $\left(\bar{\alpha}_{1} \ldots, \bar{\alpha}_{t}\right)$. Assume, for $m \geq 1$, that $P_{m} \cong P\left(b_{1}\right) \oplus P\left(b_{2}\right) \oplus \cdots \oplus P\left(b_{s}\right)$ with $s \geq 1$ and $P_{m-1} \cong P\left(a_{1}\right) \oplus P\left(a_{2}\right) \oplus \cdots \oplus P\left(a_{r}\right)$ with $r \geq 1$, where the $a_{i}, b_{j}$ are vertices with 
$a_{1}=b_{1}=a$, while $\phi_{m}$ is isomorphic to the map represented by a matrix of the following form :

$$
\left(\begin{array}{cccc}
\bar{x}_{11} & \bar{x}_{12} & \cdots & \bar{x}_{1 s} \\
0 & \bar{x}_{22} & \cdots & \bar{x}_{2 s} \\
\vdots & \vdots & \ddots & \vdots \\
0 & \bar{x}_{r 2} & \cdots & \bar{x}_{r s}
\end{array}\right),
$$

where $x_{i j}$ is a linear combination of non-trivial paths from $a_{i}$ to $b_{j}$ with $x_{11}$ being $\alpha$ or $\alpha^{n-1}$. We shall show that $\phi_{m+1}$ is isomorphic to a map represented by a matrix of this form. By Lemma 1.1, it suffices to prove that ker $\phi_{m}$ contains a top element of the form $\left(\bar{y}_{11}, 0, \ldots, 0\right)^{T}$ with $y_{11}=\alpha$ or $\alpha^{n-1}$. Indeed, if $x_{11}=\alpha^{n-1}$, then $(\bar{\alpha}, 0, \ldots, 0)^{T}$ clearly lies in ker $\phi_{m}$ but not in its radical since ker $\phi_{m} \subseteq \operatorname{rad} P_{m}$. If $x_{11}=\alpha$, then $Z=\left(\bar{\alpha}^{n-1}, 0, \ldots, 0\right)^{T} \in \operatorname{ker} \phi_{m}$. Assume on the contrary that $Z$ lies in the radical of $\operatorname{ker} \phi_{m}$. Then $Z=Y \bar{u}$ with $Y \in \operatorname{ker} \phi_{m}$ and $u \in Q^{+}$. Since $Y \in$ $\operatorname{rad} P_{m}$, we may write $Y=\left(\bar{y}_{1}, \bar{y}_{2}, \ldots, \bar{y}_{s}\right)^{T}$ with $y_{i} \in Q^{+}$. Now $v=\alpha^{n-1}-y_{1} u, y_{2} u$, $\ldots, y_{s} u \in I$ since $Z=Y \bar{u}$, and $w=\alpha y_{1}+x_{12} y_{2}+\cdots+x_{1 s} y_{s} \in I$ since $Y \in \operatorname{ker} \phi_{m}$. Therefore, $\alpha^{n}=\alpha v+\alpha y_{1} u=\alpha v+w u-\left(x_{12} y_{2} u+\cdots+x_{1 s} y_{s} u\right) \in Q^{+} I+I Q^{+}$, a contradiction. Thus $Z$ is indeed a top element of $\operatorname{ker} \phi_{m}$. By induction, we have shown that $P(a)$ is a direct summand of $P_{m}$ for all $m \geq 1$. This completes the proof of the proposition.

We now deduce some useful consequences from the above result.

1.3. Corollary. Let $A$ be the algebra of a bound quiver $(Q, I)$, containing a loop $\alpha$ at a vertex $a$. If no power of $\alpha$ is a component of a polynomial relation, then for all $i \geq 1, \operatorname{Ext}_{A}^{i}(S(a), S(a))$ does not vanish.

Proof. Let $n \geq 2$ be minimal such that $\alpha^{n} \in I$, and assume that $\alpha^{n-1}$ is not a component of any polynomial relation. Note that for a relation $\rho$ on $Q$ and $x, y \in$ $k Q, x \rho y$ is either zero or a sum of relations on $Q$. Therefore, if $\alpha^{n} \in Q^{+} I+I Q^{+}$, then

$$
\alpha^{n}=\left(\beta_{1} \rho_{1}+\cdots+\beta_{r} \rho_{r}\right)+\left(\rho_{r+1} \beta_{r+1}+\cdots+\rho_{s} \beta_{s}\right),
$$

where the $\rho_{i}$ are relations on $Q$ and the $\beta_{i}$ are arrows. Hence, $\alpha^{n-1}$ is a component of at least one of the $\rho_{i}$, say $\rho_{1}$. By the minimality of $n, \rho_{1}$ is a polynomial relation, a contradiction. The proof is now completed by applying Proposition 1.2.

The above result implies immediately the following.

1.4. Corollary. Let $A$ be the algebra of a bound quiver $(Q, I)$, containing a loop $\alpha$ at a vertex a. If $\alpha^{2} \in I$, then $\operatorname{Ext}_{A}^{i}(S(a), S(a))$ does not vanish for every $i \geq 1$.

We shall now study simple modules with an almost split self-extension, that is, invariant under the Auslander-Reiten translation $\tau=\mathrm{DTr}$; see [1, (IV.1)]. For this purpose, we need the following result, which is interesting in its own right.

1.5. Proposition. Let $A$ be the algebra of a bound quiver $(Q, I)$, and let $a, b$ be vertices of $Q$. Then $\tau S(a) \cong S(b)$ if and only if $Q$ contains an arrow $\alpha: a \rightarrow b$ which is the only arrow starting at $a$ and the only one ending at $b$.

Proof. If $Q$ satisfies the stated property, then we have a non-split exact sequence:

$$
0 \longrightarrow S(b) \longrightarrow M(\alpha) \longrightarrow S(a) \longrightarrow 0 \text {. }
$$


Since $\alpha$ is the only arrow starting at $a$ and the only one ending at $b$, one verifies easily that the sequence is almost split. Conversely assume that $S(b) \cong \tau S(a)$. Let $\alpha_{i}: a \rightarrow b_{i}, i=1, \ldots, r$, be the arrows starting at $a$; and $\beta_{j}: a_{j} \rightarrow b, j=1, \ldots, s$, be those ending at $b$. Then $S(a)$ has a minimal projective presentation

$$
\delta: \quad P\left(b_{1}\right) \oplus \cdots \oplus P\left(b_{r}\right) \longrightarrow P(a) \longrightarrow S(a) \longrightarrow 0 .
$$

Applying the duality $\operatorname{Hom}_{A}\left(-, A_{A}\right)$ to $\delta$, we get a minimal projective presentation

$$
\delta^{*}: \quad Q(a) \longrightarrow Q\left(b_{1}\right) \oplus \cdots \oplus Q\left(b_{r}\right) \longrightarrow \operatorname{Tr} S(a) \longrightarrow 0
$$

of the transpose of $S(a)$, where $T(c)$ and $Q(c)$ denote, respectively, the simple and the indecomposable projective left $A$-module associated to a vertex $c$. On the other hand, $T(b)$ has a minimal projective presentation

$$
\eta: \quad Q\left(a_{1}\right) \oplus \cdots \oplus Q\left(a_{s}\right) \longrightarrow Q(b) \longrightarrow T(b) \longrightarrow 0 .
$$

Noting $\operatorname{Tr} S(a) \cong \mathrm{D} S(b) \cong T(b)$, we have $\eta \cong \delta^{*}$. In particular, $r=1$ with $b_{1}=b$ and $s=1$ with $a=a_{1}$. The proof of the proposition is completed.

We call a module homogeneous if it admits an almost split self-extension.

1.6. Corollary. Let $A$ be the algebra of a connected bound quiver $(Q, I)$. Then $A$ admits a homogeneous simple module if and only if $Q$ consists of one loop. In this case, for any indecomposable non-projective A-module $M, \operatorname{Ext}_{A}^{i}(M, M)$ does not vanish for every $i \geq 1$.

Proof. By Proposition 1.5, $Q$ has a vertex $a$ such that $\tau S(a) \cong S(a)$ if and only if $Q$ contains a loop $\alpha: a \rightarrow a$ which is the only arrow starting at $a$ and the only one ending at $a$. This is equivalent to $Q$ consisting of one loop since $Q$ is connected. The rest of the statement is well known. This completes the proof of the corollary.

An Artin algebra is called a Nakayama algebra if its indecomposable modules are all uniserial. The algebra of a connected bound quiver is a Nakayama algebra if and only if the quiver is a single path (maybe trivial) or an oriented cycle. The following is another characterization of this class of algebras; compare [1, (IV.2.10)].

1.7. Theorem. Let $A$ be the algebra of a connected bound quiver $(Q, I)$. Then $A$ is a Nakayama algebra if and only if there exists a $\tau$-orbit $\mathcal{O}$ consisting of simple $A$-modules. In this case, $\mathcal{O}$ contains all simple A-modules.

Proof. If $Q$ is a path $a_{1} \rightarrow \cdots \rightarrow a_{n}$ with $n \geq 1$ and the $a_{i}$ pairwise distinct, by Proposition 1.5, $\tau S\left(a_{i}\right)=S\left(a_{i+1}\right)$ for $1 \leq i<n$. Since $S\left(a_{n}\right)$ is projective and $S\left(a_{1}\right)$ is injective, the $S\left(a_{i}\right)$ form a $\tau$-orbit. If $Q$ is an oriented cycle $a_{1} \rightarrow \cdots \rightarrow a_{n} \rightarrow a_{1}$ with $n \geq 1$ and the $a_{i}$ pairwise distinct, then $\tau S\left(a_{i}\right)=S\left(a_{i+1}\right)$ for $1 \leq i<n$, and $\tau S\left(a_{n}\right)=S\left(a_{1}\right)$. Hence the $S\left(a_{i}\right)$ form a $\tau$-orbit.

Conversely, let $\mathcal{O}=\left\{S\left(a_{1}\right), \ldots, S\left(a_{n}\right)\right\}$ be a $\tau$-orbit with the $a_{i}$ pairwise distinct vertices of $Q$. Consider first the case $n=1$. If $S\left(a_{1}\right)$ is projective, then $Q$ consists of the vertex $a_{1}$. Otherwise, $\tau S\left(a_{1}\right)=S\left(a_{1}\right)$, and hence $Q$ consists of one loop by Corollary 1.6. Assume now that $n>1$ and that $\tau S\left(a_{i}\right)=S\left(a_{i+1}\right)$ for $1 \leq i<n$. For each $1 \leq i<n$, by Proposition 1.5, $Q$ contains an arrow $\alpha_{i}: a_{i} \rightarrow a_{i+1}$, the only one starting at $a_{i}$ and the only one ending at $a_{i+1}$. If $S\left(a_{n}\right)$ is projective, then $S\left(a_{1}\right)$ is injective. Hence $Q$ contains no arrow starting at $a_{n}$ or ending at $a_{1}$. Thus $Q$ consists of the path $a_{1} \rightarrow \cdots \rightarrow a_{n-1} \rightarrow a_{n}$. Otherwise, $\tau S\left(a_{n}\right)=S\left(a_{1}\right)$. Then $Q$ contains an arrow $\alpha_{n}: a_{n} \rightarrow a_{1}$, the only one starting at $a_{n}$ and the only one 
ending at $a_{1}$. Thus $Q$ consists of the oriented cycle $a_{1} \rightarrow \cdots \rightarrow a_{n} \rightarrow a_{1}$. The proof of the theorem is completed.

\section{Special Biserial algebras}

The objective of this section is to establish the conjecture for special biserial algebras. Recall that a finite-dimensional $k$-algebra is called special biserial if it is isomorphic to the algebra of a bound quiver $(Q, I)$ satisfying (1) each vertex is the start-point of at most two arrows and the end-point of at most two arrows; and (2) for each arrow $\beta$, there exists at most one arrow $\alpha$ such that $\alpha \beta \notin I$ and at most one arrow $\gamma$ such that $\beta \gamma \notin I$. We call such a bound quiver special biserial.

Let $(Q, I)$ be a special biserial bound quiver. Then a relation on $Q$ is either monomial or binomial; see [9]. Moreover, if a vertex is the start-point of a binomial relation, then such a relation is unique up to a scalar. Thus we may assume, without changing its algebra, that every binomial relation of $(Q, I)$ is a multiple of a binomial relation of the form $p-q$. In this case, we call $(p, q)$ a binomial pair.

For a vertex $a$, the trivial path at $a$ is denoted by $\varepsilon_{a}$, and for a path $p$, its startpoint and end-point are denoted by $s(p)$ and $e(p)$, respectively. For an arrow $\alpha$, we introduce a new arrow $\alpha^{-1}$, the inverse of $\alpha$ with $s\left(\alpha^{-1}\right)=e(\alpha)$ and $e\left(\alpha^{-1}\right)=s(\alpha)$. A reduced walk $w$ in $Q$ is either a trivial path or $w=c_{1} c_{2} \cdots c_{n}$ with $n \geq 1$, where $c_{i}$ is either an arrow or the inverse of an arrow such that $s\left(c_{i+1}\right)=e\left(c_{i}\right)$ and $c_{i+1} \neq c_{i}^{-1}$ for all $1 \leq i<n$. In the latter case, a path $p=\alpha_{1} \cdots \alpha_{r}$ with $\alpha_{i}$ arrows is contained in $w$ if there exists $i$ with $1 \leq i \leq n$ such that either $c_{i+j}=\alpha_{j+1}$ for all $0 \leq j<r$ or $c_{i+j}=\alpha_{r-j}^{-1}$ for all $0 \leq j<r$. A reduced walk $w$ is a string if no path contained in $w$ is a component of a relation on $Q$. A string $p$ is called serial if it is a path and $s(p)$ is not the start-point of any binomial relation. Finally, we say that a string $w$ starts or ends in a deep if there is no arrow $\gamma$ such that $\gamma^{-1} w$ or $w \gamma$ is a string, respectively.

Let $A=k Q / I$. To each string $w$, one associates a string module $M(w)$, compare 3, 10, as follows: if $w=\varepsilon_{a}$ for a vertex $a$, then $M(w)=S(a)$ with $\{a\}$ a $k$-basis. If $w=c_{1} c_{2} \cdots c_{n}$ with $c_{i}$ an arrow or the inverse of an arrow, then $M(w)$ has as a $k$-basis the ordered family $\left\{a_{0}, a_{1}, \ldots, a_{n}\right\}$, where $a_{0}=s\left(c_{1}\right)$ and $a_{i}=e\left(c_{i}\right)$ for $1 \leq i \leq n$. Its multiplication is such that for an arrow $\alpha$, one has $a_{i} \bar{\alpha}=a_{i+1}$ if $c_{i+1}=\alpha$ with $0 \leq i<n$, and $a_{i} \bar{\alpha}=a_{i-1}$ if $c_{i}=\alpha^{-1}$ with $1 \leq i \leq n$, and $a_{i} \bar{\alpha}=0$ otherwise. Here $a_{i}$ with $0 \leq i \leq n$ is a top element if and only if $c_{i}$ is the inverse of an arrow whenever $i \geq 1$, and $c_{i+1}$ is an arrow whenever $i<n$.

Furthermore, for a vertex $a$ of $Q$, we shall fix a $k$-basis of $P(a)$ with a multiplication. If $\varepsilon_{a}$ is serial, then there exist paths $p, q$ starting at $a$ such that $p^{-1} q$ is a string starting and ending in a deep. In this case, $P(a)=M\left(p^{-1} q\right)$. Otherwise, there exists a binomial relation $\alpha_{1} \alpha_{2} \cdots \alpha_{r}-\beta_{1} \beta_{2} \cdots \beta_{s}$, where $\alpha_{i}, \beta_{j}$ are arrows with $s\left(\alpha_{1}\right)=s\left(\beta_{1}\right)=a$. Then $P(a)$ has as a $k$-basis the ordered family $\left\{a_{1}, \ldots, a_{r}, b_{1}, \ldots, b_{s}\right\}$, where $a_{i}=s\left(\alpha_{i}\right)$ and $b_{j}=e\left(\beta_{j}\right)$. Its multiplication is such that for each arrow $\alpha$, one has $b_{j} \bar{\alpha}=b_{j+1}$ if $\alpha=\beta_{j+1}$ with $1 \leq j<s$ and $b_{j} \bar{\alpha}=0$ otherwise; moreover,

$$
a_{i} \bar{\alpha}= \begin{cases}b_{1}, & i=1 \text { and } \alpha=\beta_{1} \\ a_{i+1}, & 1 \leq i<r \text { and } \alpha=\alpha_{i}, \\ b_{s}, & i=r \text { and } \alpha=\alpha_{r} \\ 0, & \text { otherwise }\end{cases}
$$


Here $a_{1}$ is the only top element. In all cases, we call the fixed $k$-basis of $M(w)$ or $P(a)$ its canonical basis. Clearly, the classes of the top elements of the canonical basis form a basis for the top in each case.

In order to describe the syzygies of string modules, we need the notion of syzygy strings of a string, defined in the following.

2.1. Definition. Let $(Q, I)$ be a special biserial bound quiver. Let $w$ be a string, and write

$$
w=p_{1}^{-1} q_{1} \cdots p_{r}^{-1} q_{r},
$$

where $r \geq 1$, the $p_{i}, q_{i}$ are paths with $p_{1}, q_{r}$ the only ones that may be trivial.

(1) In the case that $p_{1}$ is non-serial, we let $\alpha_{1}$ be an arrow and $u_{1}, v_{1}$ some paths such that $\left(p_{1} \alpha_{1} u_{1}, q_{1} v_{1}\right)$ is a binomial pair. Assume now that $p_{1}$ is serial. We first define $v_{1}$ to be a path such that $q_{1} v_{1}$ is a string ending in a deep. Moreover, if $p_{1}^{-1} q_{1}$ does not start in a deep, then let $\alpha_{1}$ be an arrow and $u_{1}$ a path such that $u_{1}^{-1} \alpha_{1}^{-1} p_{1}^{-1} q_{1}$ is a string starting in a deep, and we define neither $\alpha_{1}$ nor $u_{1}$ otherwise.

(2) Now let $i$ be an integer with $1<i<r$. If $p_{i}$ is non-serial, then let $u_{i}, v_{i}$ be the paths such that $\left(p_{i} u_{i}, q_{i} v_{i}\right)$ is a binomial pair. Otherwise, let $u_{i}, v_{i}$ be paths such that $p_{i} u_{i}$ and $q_{i} v_{i}$ are strings ending in a deep.

(3) In the case that $p_{r}$ is non-serial, let $\alpha_{r}$ be an arrow and $u_{r}, v_{r}$ some paths such that $\left(p_{r} u_{r}, q_{r} \alpha_{r} v_{r}\right)$ is a binomial pair. Assume now that $p_{r}$ is serial. First let $u_{r}$ be a path such that $p_{r} u_{r}$ is a string ending in a deep. Moreover, if $p_{r}^{-1} q_{r}$ does not end in a deep, then let $\alpha_{r}$ be an arrow and $v_{r}$ some path such that $p_{r}^{-1} q_{r} \alpha_{r} v_{r}$ is a string ending in a deep; we define neither $\alpha_{r}$ nor $v_{r}$ otherwise.

For all $1 \leq i<r$, let $w_{i}=v_{i}^{-1} u_{i+1}$, which is clearly a string. Moreover, let $w_{0}=u_{1}$ if $u_{1}$ is defined, and $w_{r}=v_{r}^{-1}$ if $v_{r}$ is defined. Denote by $W$ the set of defined strings $w_{i}$ with $0 \leq i \leq r$. We say that $w_{s}, w_{t}$ with $0 \leq s<t \leq r$ are connected if $p_{i}$ is non-serial for all $s<i \leq t$. This relation of connectedness generates an equivalence relation on $W$. It is easy to see that if $\left\{w_{i}, w_{i+1}, \ldots, w_{j}\right\}$ with $0 \leq i \leq j \leq r$ is an equivalence class of $W$, then $w_{i} w_{i+1} \cdots w_{j}$ is a string, called a syzygy string of $w$. Note that the number of the syzygy strings of $w$ is equal to the number of the equivalence classes of $W$.

The following is the promised combinatorial description of the first syzygy of a string module.

2.2. Proposition. Let $A=k Q / I$ with $(Q, I)$ special biserial. Let $w$ be a string and $\Omega(w)$ the set of the syzygy strings of $w$. If $M(w)$ is the string module associated to $w$, then $\Omega(M(w))=\bigoplus_{\omega \in \Omega(w)} M(\omega)$.

Proof. We keep all the notation introduced in Definition 2.1. Let $a_{i}=s\left(p_{i}\right), i=$ $1, \ldots, r$, be the top elements of the canonical basis of $M(w)$. Then a projective cover of $M(w)$ is given by the map $\phi: P\left(a_{1}\right) \oplus \cdots \oplus P\left(a_{r}\right) \rightarrow M(w)$ such that the top element of the canonical basis of $P\left(a_{i}\right)$ maps to that of $M\left(p_{i}^{-1} q_{i}\right)$ for all $1 \leq i \leq r$. It is easy to see that $M(w)$ is projective if and only if $\Omega(w)$ is empty. Assume that $\Omega(w)$ is non-empty. Since the result is obvious for the case where $r=1$, we may assume furthermore that $r>1$.

In order to describe the kernel of $\phi$, we need more notation. If $u_{1}$ is defined and it is non-trivial whenever $p_{1}$ is non-serial, then let $b_{1}$ be the element of the canonical basis of $P\left(a_{1}\right)$ corresponding to $s\left(u_{1}\right)$ and let $d_{0}$ be the top element of the canonical basis of $M\left(w_{0}\right)$. Otherwise, we define neither $b_{1}$ nor $d_{0}$. In any case, 
let $c_{1}$ be the element of the canonical basis of $P\left(a_{1}\right)$ corresponding to $e\left(q_{1}\right)$, and let $d_{1}$ be the top element of $M\left(w_{1}\right)$. For each $1<i<r$, let $b_{i}$ and $c_{i}$ be the elements of the canonical basis of $P\left(a_{i}\right)$ corresponding to $e\left(p_{i}\right)$ and $e\left(q_{i}\right)$, respectively, and let $d_{i}$ be the top element of the canonical basis of $M\left(w_{i}\right)$. Finally, if $v_{r}$ is defined and it is non-trivial whenever $q_{r}$ is non-serial, then let $c_{r}$ be the element of the canonical basis of $P\left(a_{r}\right)$ corresponding to $e\left(\alpha_{r}\right)$, and let $d_{r}$ be the top element of the canonical basis of $M\left(w_{r}\right)$. Otherwise, we define neither $c_{r}$ nor $d_{r}$. In any case, let $b_{r}$ be the element of the canonical basis of $P\left(a_{r}\right)$ corresponding to $e\left(p_{r}\right)$.

Write $\hat{d}_{i}=\left(0, \ldots, 0, d_{i}, 0, \ldots, 0\right)^{T} \in \bigoplus_{\omega \in \Omega(w)} M(\omega)$ if $d_{i}$ is defined. Then the classes of the defined $\hat{d}_{i}$ with $0 \leq i \leq r$ form a $k$-basis of the top of $\bigoplus_{\omega \in \Omega(w)} M(\omega)$. Let $\psi: \bigoplus_{\omega \in \Omega(w)} M(\omega) \rightarrow P\left(a_{1}\right) \oplus \cdots \oplus P\left(a_{r}\right)$ be the map such that

$$
\psi\left(\hat{d}_{i}\right)= \begin{cases}\left(b_{1}, 0, \ldots, 0\right)^{T}, & i=0 \text { and } d_{0} \text { is defined } \\ \left(0, \ldots, 0,-c_{i}, b_{i+1}, 0, \ldots, 0\right)^{T}, & 1 \leq i<r \\ \left(0, \ldots, 0, c_{r}\right)^{T}, & i=r \text { and } d_{r} \text { is defined }\end{cases}
$$

One easily verifies that $\psi$ is a monomorphism such that $\psi \phi=0$. By calculating the dimensions of the modules, we deduce finally that $\psi$ is the kernel of $\phi$. This completes the proof of the proposition.

By Corollary 1.3, we need consider only special biserial bound quivers with a loop such that at least one of its powers is a component of a binomial relation. For convenience, we make the following two definitions.

2.3. Definition. Let $(Q, I)$ be a special biserial bound quiver, containing a loop $\alpha$ at a vertex $a$ and a binomial pair $\left(\alpha^{n+1}, \alpha_{1} \cdots \alpha_{m}\right)$ with $n \geq 1$ and $m \geq 2$. We shall consider strings of the following forms:

(1) The trivial string $\varepsilon_{a}$.

(2) The string

$$
q_{r+1} q_{r}^{-1} \cdots q_{2} q_{1}^{-1} \alpha p_{1}^{-1} p_{2} \cdots p_{r}^{-1} p_{r+1}
$$

where $r \geq 1$ is odd, the $p_{i}, q_{i}$ are paths that are non-trivial for $1 \leq i \leq r$ such that $\left(p_{i} q_{i}, p_{i+1} q_{i+1}\right)$ is a binomial pair for each odd $i$ with $1 \leq i<r$, and there exists a binomial pair $\left(p_{r} q_{r}, p_{r+1} \beta_{r+1} q_{r+1}\right)$ with $\beta_{r+1}$ an arrow.

(3) The string

$$
q_{s}^{-1} q_{s-1} \cdots q_{2} q_{1}^{-1} \alpha p_{1}^{-1} p_{2} \cdots p_{s-1} p_{s}^{-1} \cdots p_{r}^{-1} p_{r+1},
$$

where $s, r$ are odd with $1 \leq s \leq r$, the $p_{i}, q_{j}$ are paths that are non-trivial for $1 \leq i \leq r$ and $1 \leq j<s$ such that $\left(p_{i} q_{i}, p_{i+1} q_{i+1}\right)$ is a binomial pair for each odd $i$ with $1 \leq i<s$, and $p_{s} q_{s}$ is a serial string ending in a deep.

2.4. Definition. Let $(Q, I)$ be as in Definition 2.3. We shall consider strings of the following forms:

(1) The string

$$
p_{r+1} p_{r}^{-1} \cdots p_{2}^{-1} p_{1} \alpha^{-n} q_{1} q_{2}^{-1} \cdots q_{r}^{-1} q_{r+1},
$$

where $r \geq 0$ is even, the $p_{i}, q_{i}$ are paths that are non-trivial for $1 \leq i \leq r$ such that $\left(q_{i} p_{i}, q_{i+1} p_{i+1}\right)$ is a binomial pair for each even $0 \leq i<r$ and there exists a binomial pair $\left(q_{r} p_{r}, q_{r+1} \beta_{r+1} p_{r+1}\right)$ with $\beta_{r+1}$ an arrow, $q_{0}=\alpha^{n}$ and $p_{0}=\alpha$.

(2) The string

$$
p_{s}^{-1} p_{s-1} \cdots p_{2}^{-1} p_{1} \alpha^{-n} q_{1} q_{2}^{-1} \cdots q_{s-1} q_{s}^{-1} \cdots q_{r}^{-1} q_{r+1},
$$


where $s, r$ are even with $2 \leq s \leq r$, the $p_{i}, q_{j}$ are paths that are non-trivial for $1 \leq i<s$ and $1 \leq j \leq r$, such that $\left(q_{i} p_{i}, q_{i+1} p_{i+1}\right)$ is a binomial pair for each even $i$ with $2 \leq i<s$, and $q_{s} p_{s}$ is a serial string ending in a deep.

We shall now apply Proposition 2.2 to describe the first syzygy of string modules associated to previously defined strings.

2.5. Lemma. Let $A=k Q / I$ with $(Q, I)$ being as in Definition 2.3. If $M$ is a string module associated to a string as stated in Definition 2.3 , then $\Omega(M)$ has as a direct summand a string module associated to a string as stated in Definition 2.4.

Proof. Let $M=M(w)$ with $w$ a string. First, if $w=\varepsilon_{a}$, then $M=S(a)$. Thus $\Omega(M)$ is the string module associated to the string $\left(\alpha_{2} \cdots \alpha_{m}\right) \alpha^{-n}$, which is of the form stated in Definition 2.4(1) with $r=0$ and $q_{1}$ trivial. Second, we consider the case

$$
w=q_{r+1} q_{r}^{-1} \cdots q_{2} q_{1}^{-1} \alpha p_{1}^{-1} p_{2} \cdots p_{r}^{-1} p_{r+1}
$$

where $r \geq 1$ is odd, and the $p_{i}, q_{i}$ are non-trivial paths such that $\left(p_{i} q_{i}, p_{i+1} q_{i+1}\right)$ is a binomial pair for each odd $i$ with $1 \leq i<r$, and there exists a binomial pair $\left(p_{r} q_{r}, p_{r+1} \beta_{r+1} q_{r+1}\right)$ with $\beta_{r+1}$ an arrow. Note that $q_{1}=\alpha_{1} \cdots \alpha_{t}$ with $1 \leq t<m$.

Suppose first that $q_{r+1}$ is trivial and that there exists no even $i$ with $2 \leq i<r$ such that $q_{i}$ is serial. If $r=1$, then let $u_{1}$ be the path (maybe trivial) such that $\alpha_{t+1} \cdots \alpha_{m}=\alpha_{t+1} u_{1}$. Note that we have binomial pairs $\left(q_{1} \alpha_{t+1} u_{1}, \alpha \alpha^{n}\right)$ and $\left(p_{1} q_{1}, p_{2} \beta_{2}\right)$. By Proposition 2.2, $\Omega(M)$ is the string module associated to the string $u_{1} \alpha^{-n} q_{1}$, which is of the form stated in Definition 2.4(1). If $r \geq 3$, let $u_{1}=\alpha_{t+1} \cdots \alpha_{m}$, and for each even $i$ with $2 \leq i \leq r-3$, let $u_{i}, u_{i+1}$ be non-trivial paths such that $\left(q_{i} u_{i}, q_{i+1} u_{i+1}\right)$ is a binomial pair, and finally, let $u_{r}, u_{r-1}$ be paths with $u_{r-1}$ non-trivial and $\delta_{r}$ an arrow such that $\left(q_{r-1} u_{r-1}, q_{r} \delta_{r} u_{r}\right)$ is a binomial pair. Since none of the paths contained in $w$ is a serial string, $\Omega(M)$ is the string module associated to the string $u_{r} u_{r-1}^{-1} \cdots u_{3} u_{2}^{-1} u_{1} \alpha^{-n} q_{1} q_{2}^{-1} q_{3} \cdots q_{r-1}^{-1} q_{r}$, which is of the form stated in Definition 2.4(1) for $r-1$.

Suppose now that $q_{r+1}$ is non-trivial and that there exists no even $i$ with $2 \leq$ $i \leq r+1$ such that $q_{i}$ is serial. Let $u_{1}=\alpha_{t+1} \cdots \alpha_{m}$ and let, for each even $i$ with $2 \leq i \leq r-1, u_{i}, u_{i+1}$ be non-trivial paths such that $\left(q_{i} u_{i}, q_{i+1} u_{i+1}\right)$ is a binomial pair, and finally, let $u_{r+2}, u_{r+1}$ be non-trivial paths and $\delta_{r+2}$ an arrow such that $\left(q_{r+1} u_{r+1}, \delta_{r+2} u_{r+2}\right)$ is a binomial pair. For the same reason, $\Omega(M)$ is the string module associated to the string $u_{r+2} u_{r+1}^{-1} \cdots u_{2}^{-1} u_{1} \alpha^{-n} q_{1} q_{2}^{-1} \cdots q_{r} q_{r+1}^{-1}$, which is of the form stated in Definition 2.4(1) for $r+1$ with $q_{r+2}$ trivial.

Suppose that neither of the above two situations occurs. Then there exists some minimal even integer $s$ with $2 \leq s \leq r+1$ such that $q_{s}$ is non-trivial and serial. Let $u_{1}=\alpha_{t+1} \cdots \alpha_{m}$ and let, for each even $i$ with $2 \leq i<s, u_{i}, u_{i+1}$ be non-trivial paths such that $\left(q_{i} u_{i}, q_{i+1} u_{i+1}\right)$ is a binomial pair, and finally, let $u_{s}$ be a path (maybe trivial) such that $q_{s} u_{s}$ is a string ending in a deep. Since the $p_{i}, q_{j}$ with $1 \leq i \leq r$ and $1 \leq j<s$ are non-serial, $\Omega(M)$ has as a direct summand the string module associated to the string $u_{s}^{-1} u_{s-1} \cdots u_{2}^{-1} u_{1} \alpha^{-n} q_{1} q_{2}^{-1} \cdots q_{s-1} q_{s}^{-1} \cdots q_{r} q_{r+1}^{-1}$, which is of the form stated in Definition 2.4(2) for $s$ and $r-1$ if $s \leq r-1$ and $p_{r+1}$ is trivial, and for $s$ and $r+1$ otherwise.

We conclude the proof with the final case:

$$
w=q_{s}^{-1} q_{s-1} \cdots q_{2} q_{1}^{-1} \alpha p_{1}^{-1} p_{2} \cdots p_{s-1} p_{s}^{-1} \cdots p_{r}^{-1} p_{r+1}
$$

where $s, r$ are odd with $1 \leq s \leq r$, and the $p_{i}, q_{j}$ are non-trivial paths for $1 \leq i \leq r$ and $1 \leq j<s$ such that $\left(p_{i} q_{i}, p_{i+1} q_{i+1}\right)$ is a binomial pair for each odd $i$ with 
$1 \leq i<s$, and $p_{s} q_{s}$ is a serial string ending in a deep. Suppose first that $q_{i}$ is nonserial for each odd $i$ with $1 \leq i \leq s$. Let $u_{s}, u_{s-1}$ be paths with $u_{s-1}$ non-trivial and $\delta_{s}$ an arrow such that $\left(q_{s} \delta_{s} u_{s}, q_{s-1} u_{s-1}\right)$ is a binomial pair, where $q_{0}=\alpha$ and $u_{0}=\alpha^{-n}$ in case $s=1$; and let $u_{1}=\alpha_{t+1} \cdots \alpha_{m}$ if $s>1$. Finally for each odd $i$ with $3 \leq i<s$, let $u_{i}, u_{i-1}$ be non-trivial paths such that $\left(q_{i-1} u_{i-1}, q_{i} u_{i}\right)$ is a binomial pair. Since $p_{s}$ is serial while the $p_{i}, q_{j}$ with $1 \leq i<s$ and $1 \leq j \leq s$ are non-serial, $\Omega(M)$ has as a direct summand the string module associated to the string $u_{s} u_{s-1}^{-1} \cdots u_{1} \alpha^{-n} q_{1} q_{2}^{-1} \cdots q_{s-1}^{-1} q_{s}$, which is of the form stated in Definition 2.4(1) for $s-1$. Otherwise $s \geq 3$ and there exists a minimal even integer $d$ with $2 \leq d \leq s-1$ such that $q_{d}$ is serial. Let $u_{d}$ be a path (maybe trivial) such that $q_{d} u_{d}$ is a string ending in a deep. Being non-trivial, $q_{1}=\alpha_{1} \cdots \alpha_{t}$ for some $1 \leq t<m$. Let $u_{1}=\alpha_{t+1} \cdots \alpha_{m}$, and for each even $i$ with $2 \leq i<d$, let $u_{i}, u_{i+1}$ be non-trivial paths such that $\left(q_{i} u_{i}, q_{i+1} u_{i+1}\right)$ is a binomial pair. Since $q_{d}$ and $p_{s}$ are serial while the others between them are non-serial, $\Omega(M)$ has as a direct summand the string module associated to the string $u_{d}^{-1} u_{d-1} \cdots u_{2}^{-1} u_{1} \alpha^{-n} q_{1} q_{2}^{-1} \cdots q_{s-1}^{-1} q_{s}$, which is of the form stated in Definition 2.4(2) for $d$ and $s-1$. This completes the proof.

2.6. Lemma. Let $A=k Q / I$ with $(Q, I)$ being as in Definition 2.3. If $M$ is a string module associated to a string as stated in Definition 2.4, then $\Omega(M)$ has as a direct summand a string module associated to a string as stated in Definition 2.3.

Proof. Let $M=M(w)$ with $w$ a string. Let us begin with the case

$$
w=p_{r+1} p_{r}^{-1} \cdots p_{2}^{-1} p_{1} \alpha^{-n} q_{1} q_{2}^{-1} \cdots q_{r}^{-1} q_{r+1},
$$

where $r \geq 0$ is even, the $p_{i}, q_{i}$ are non-trivial for $1 \leq i \leq r$ such that for each even $i$ with $0 \leq i<r,\left(q_{i} p_{i}, q_{i+1} p_{i+1}\right)$ is a binomial pair with $q_{0}=\alpha^{n}$ and $p_{0}=\alpha$, and there exists a binomial pair $\left(q_{r} p_{r}, q_{r+1} \beta_{r+1} p_{r+1}\right)$ with $\beta_{r+1}$ some arrow.

Suppose first that $p_{r+1}$ is trivial and that there exists no odd $i$ with $1 \leq i<$ $r$ such that $p_{i}$ is serial. If $r=0$, then $q_{1}=\alpha_{1} \cdots \alpha_{m-1}$ by hypothesis. Thus $\Omega(M)=M\left(\varepsilon_{a}\right)$. If $r \geq 2$, then $p_{1}=\alpha_{t} \cdots \alpha_{m}$ with $1<t \leq m$. For each odd $i$ with $1 \leq i \leq r-3$, let $u_{i}, u_{i+1}$ be non-trivial paths such that $\left(p_{i} u_{i}, p_{i+1} u_{i+1}\right)$ is a binomial pair, and let $\delta_{r}$ be an arrow and $u_{r}, u_{r-1}$ some paths with $u_{r-1}$ nontrivial such that $\left(q_{r-1} u_{r-1}, q_{r} \delta_{r} u_{r}\right)$ is a binomial pair. Since none of the paths contained in $w$ is a serial string, $\Omega(M)$ is the string module associated to the string $u_{r} u_{r-1}^{-1} \cdots u_{2} u_{1}^{-1} \alpha p_{1}^{-1} p_{2} \cdots p_{r-1}^{-1} p_{r}$, which is of the form stated in Definition 2.3(1) for $r-1$.

Suppose secondly that $p_{r+1}$ is non-trivial and that there exists no odd $i$ with $1 \leq i \leq r+1$ such that $p_{i}$ is serial. For each odd $i$ with $1 \leq i \leq r-1$, let $u_{i}, u_{i+1}$ be non-trivial paths such that $\left(q_{i} u_{i}, q_{i+1} u_{i+1}\right)$ is a binomial pair. Moreover, let $\delta_{r+2}$ be an arrow and $u_{r+2}, u_{r+1}$ some non-trivial paths such that $\left(q_{r+1} u_{r+1}, \delta_{r+2} u_{r+2}\right)$ is a binomial pair. For the same reason, $\Omega(M)$ is the string module associated to the string $u_{r+2} u_{r+1}^{-1} \cdots u_{2} u_{1}^{-1} \alpha p_{1}^{-1} p_{2} \cdots p_{r} p_{r+1}^{-1}$, which is of the form stated in Definition 2.3(1) for $r+1$ with $p_{r+2}$ trivial.

Suppose now that neither of the above two situations occurs. Then there exists a minimal odd integer $s$ with $1 \leq s \leq r+1$ such that $p_{s}$ is non-trivial and serial. Let $u_{s}$ be a path (maybe trivial) such that $p_{s} u_{s}$ is a string ending in a deep, and for each odd $i$ with $1 \leq i<s$, let $u_{i}, u_{i+1}$ be non-trivial paths such that $\left(q_{i} u_{i}, q_{i+1} u_{i+1}\right)$ is a binomial pair. Since the $p_{i}, q_{j}$ with $1 \leq i<s$ and $1 \leq j \leq r$ are all non-serial, $\Omega(M)$ has as a direct summand the string module associated to the string $u_{s}^{-1} u_{s-1} \cdots u_{2} u_{1}^{-1} \alpha p_{1}^{-1} p_{2} \cdots p_{s-1} p_{s}^{-1} \cdots p_{r} p_{r+1}^{-1}$, which is of the form stated 
in Definition 2.3(2) for $s$ and $r-1$ if $p_{r+1}$ is trivial with $s \leq r-1$; and otherwise, for $s$ and $r+1$ with $p_{r+2}$ trivial. We shall conclude the proof with the case:

$$
w=p_{s}^{-1} p_{s-1} \cdots p_{2}^{-1} p_{1} \alpha^{-n} q_{1} q_{2}^{-1} \cdots q_{s-1} q_{s}^{-1} \cdots q_{r}^{-1} q_{r+1},
$$

where $s, r$ are even with $2 \leq s \leq r$, and the $p_{i}, q_{j}$ are non-trivial paths for $1 \leq i<s$ and $1 \leq j \leq r$ such that for each even $i$ with $0 \leq i<s,\left(q_{i} p_{i}, q_{i+1} p_{i+1}\right)$ is a binomial pair with $q_{0}=\alpha^{n}, p_{0}=\alpha$, whereas $q_{s} p_{s}$ is a serial string ending in a deep.

Suppose first that $p_{i}$ is non-serial for every odd $i$ with $1 \leq i<s$. Let $\delta_{s}$ be an arrow and $u_{s}, u_{s-1}$ some paths with $u_{s-1}$ non-trivial such that $\left(p_{s} \delta_{s} u_{s}, p_{s-1} u_{s-1}\right)$ is a binomial pair; and for each even $i$ with $2 \leq i<s$, let $u_{i}$ and $u_{i-1}$ be non-trivial paths such that $\left(q_{i-1} u_{i-1}, q_{i} u_{i}\right)$ is a binomial pair. Since $q_{s}$ is serial while the $p_{i}$, $q_{j}$ with $1 \leq i \leq s$ and $1 \leq j<s$ are all non-serial, $\Omega(M)$ has as a direct summand the string module associated to the string $u_{s} u_{s-1}^{-1} \cdots u_{1}^{-1} \alpha p_{1}^{-1} p_{2} \cdots p_{s-1}^{-1} p_{s}$, which is of the form stated in Definition 2.3(1) for $s-1$.

Otherwise, there exists a minimal odd integer $d$ with $1 \leq d \leq s-1$ such that $p_{d}$ is serial. Let $u_{d}$ be a path (maybe trivial) such that $q_{d} u_{d}$ is a string ending in a deep, and for each odd $i$ with $1 \leq i<d$, let $u_{i}, u_{i+1}$ be non-trivial paths such that $\left(q_{i} u_{i}, q_{i+1} u_{i+1}\right)$ is a binomial pair. Since $p_{d}$ and $q_{s}$ are serial while the others between them are all non-serial, $\Omega(M)$ has as a direct summand the string module associated to the string $u_{d}^{-1} u_{d-1} \cdots u_{2} u_{1}^{-1} \alpha p_{1}^{-1} p_{2} \cdots p_{s-1}^{-1} p_{s}$, which is of the form stated in Definition 2.3(2) for $d$ and $s-1$. This completes the proof of the lemma.

Let $A$ be the algebra of a bound quiver $(Q, I)$. The convex support of an $A$ module $M$ is the algebra of the bound quiver $\left(Q_{M}, I_{M}\right)$, where $Q_{M}$ is the convex hull in $Q$ of the vertices $a$ with $\operatorname{Hom}_{A}(P(a), M) \neq 0$, and $I_{M}=I \cap\left(k Q_{M}\right)$.

2.7. Theorem. Let $A$ be the algebra of a bound quiver $(Q, I)$, and let $S$ be a simple $A$-module with a non-split self-extension. If the convex support of $S$ is special biserial, then for all $i \geq 1, \operatorname{Ext}_{A}^{i}(S, S)$ does not vanish.

Proof. Let $B$ be the convex support of $S$ in $A$. It is well known that $\operatorname{Ext}_{A}^{i}(S, S)=$ $\operatorname{Ext}_{B}^{i}(S, S)$ for all $i \geq 1$. Hence we may assume, without loss of generality, that $(Q, I)$ is special biserial. Let $\alpha$ be a loop at the vertex $a$ such that $S=S(a)$. If no power of $\alpha$ is a component of a binomial relation on $Q$, then the theorem follows from Corollary 1.3. Assume now that there exists a binomial pair $\left(\alpha^{n+1}, \alpha_{1} \cdots \alpha_{m}\right)$ with $n \geq 1$ and $m \geq 2$. Note that $\Omega^{0}(S)=M\left(\varepsilon_{a}\right)$. Assume, for $i \geq 0$, that $\Omega^{i}(S)$ has as a direct summand a string module associated to a string stated in Definitions 2.3 or 2.4, and in particular, $S(a)$ is a summand of the top of $\Omega^{i}(S)$. By Lemmas 2.5 and 2.6, the same holds true for $\Omega^{i+1}(S)$. This completes the proof of the theorem.

We conclude with an even stronger version of the strong no loop conjecture.

2.8. Conjecture. If $S$ is a simple module over an $\operatorname{Artin}$ algebra $A$ with $\operatorname{Ext}_{A}^{1}(S, S)$ not vanishing, then $\operatorname{Ext}_{A}^{i}(S, S)$ does not vanish for infinitely many $i$.

\section{ACKNOWLEDGEMENTS}

Both authors are supported in part by the Natural Sciences and Engineering Research Council of Canada. 


\section{REFERENCES}

[1] M. Auslander, I. Reiten and S. O. Smalø, Representation Theory of Artin Algebras, Cambridge Studies in Advanced Mathematics 36 (Cambridge University Press, Cambridge, 1995). MR 96c:16015

[2] W. D. Burgess and M. Saoín, Homological aspects of semigroup gradings on rings and algebras, Canad. J. Math. 51(3) (1999) 488 - 505. MR 2000i:16089

[3] M. C. R. Butler and C. M. Ringel, Auslander-Reiten sequences with few middle terms and applications to string algebras, Comm. Algebra 15 (1987) 145 - 179. MR 88a:16055

[4] I. M. Gelfand and V. A. Ponomarev, Indecomposable representations of the Lorentz group, Russian Math. Surveys 23 (1968) 1 - 58. MR 37:5325

[5] E. L. Green, Ø. Solberg and D. Zacharia, Minimal projective resolutions, Trans. Amer. Math. Soc. 353 (2001), 2915-2939. MR 2002c:16010

[6] K. Igusa, Notes on the no loop conjecture, J. Pure Appl. Algebra 69(2) (1990) 161 - 176. MR 92b:16013

[7] J. P. Janusz, Indecomposable modules for finite groups, Ann. Math. 89 (1969) 209 - 241. MR 39:5622

[8] H. Lenzing, Nilpotente Elemente in Ringen von Endlicher Globaler Dimension, Math. Z. 108 (1969) 313 - 324. MR 39:1498

[9] A. Skowroński and J. Waschbüsch, Representation-finite biserial algebras, J. Reine Angew Math. 345 (1983) 172 - 181. MR 85e:16051

[10] B. Wald and J. Waschbüsch, Tame biserial algberas, J. Algebra 95 (1985) 480 - 500. MR 87a:16056

[11] D. Zacharia, Special monomial algebras of finite global dimension, NATO Adv. Sci. Inst. 233 (Kluwer, Dordrecht, 1988) 375 - 378. MR 91d:16015

Département de Mathématiques, Université de Sherbrooke, Sherbrooke, Québec, CANADA J1K 2R1

E-mail address: shiping.liu@usherbrooke.ca

Département de Mathématiques et d’Informatique, Université de Sherbrooke, SherBRooke, QuÉBec, Canada J1K 2R1

E-mail address: jphil18@hotmail.com. 